\title{
Filtro Activo adaptado a diferentes configuraciones operativas de una Planta Industrial
}

\author{
V.F. Corasaniti ${ }^{\# 1}$, M.B. Barbieri ${ }^{\# 2}$, P.L. Arnera ${ }^{\# 3}$ \\ ${ }^{\#}$ IITREE-LAT FI-UNLP (Instituto de Investigaciones Tecnológicas para Redes y Equipos Eléctricos) \\ Facultad de Ingeniería - Universidad Nacional de La Plata \\ 48 y 116. La Plata. Argentina \\ fcorasaniti@iitree-unlp.org.ar \\ 2 bbarbieri@itree-unlp.org.ar \\ pla@iitree-unlp.org.ar
}

\begin{abstract}
In this paper the analysis of a Hybrid Active Power Filter for reactive and harmonic compensation in an industrial plant is presented. This proposal uses existing capacitor banks, which are transformed into passive filters which together with an active part form an adaptive and economic compensation system.

Records of measurements at the plant were obtained, where the levels of harmonic distortion can be seen. It is analyzed different current and future operating scenarios, defined from the possible reconfiguration of the network, the possibility of changing the feed of some loads; and increasing reactive compensation with increasing future demand.

The objective of this work is to design and conveniently locate the active-passive compensation system, choosing the most economical combination that best suits the states most probable network operation. As part of the design of the control system, it is determined the measurement location of the variables of references and the connection point of the filter.
\end{abstract}

Resumen- En este trabajo se presenta el análisis de un Filtro Activo de Potencia Híbrido para la compensación de reactivo y armónicas en una planta industrial. Esta propuesta utiliza bancos de capacitores existentes, los cuales son transformados en filtros pasivos y conjuntamente con una parte activa conforman un sistema adaptable y económico.

Se poseen registros de mediciones efectuadas en dicha planta, donde se aprecian los niveles de distorsión armónica. Se analizan diferentes escenarios actuales $y$ futuros de operación, definidos a partir de la posible reconfiguración de la red, la posibilidad de cambiar el punto de alimentación de algunas cargas y el aumento de la compensación de reactivo con el incremento de la demanda futura.

El objetivo del trabajo es diseñar y ubicar convenientemente el sistema de compensación activo-pasivo, eligiendo la combinación más económica y que mejor se adapte a los estados de operación más probables de la red. Como parte del diseño del sistema de control se determina el lugar de medición de las variables de referencias y el punto de conexión del filtro.

\section{INTRODUCCIÓN}

A partir de estudios realizados en la red eléctrica correspondiente a una refinería, se poseen datos del equipamiento y resultados de mediciones realizadas en la misma. Las mediciones establecieron la existencia de tensiones y corrientes distorsionadas en distintos puntos de la red. Esto es debido a la presencia de cargas no-lineales, más precisamente de grandes motores alimentados a través de reguladores de velocidad variable (ASD o Drivers) y a los efectos de resonancias establecidas por bancos de capacitores instalados para la compensación de reactivo.
El uso de compensación tradicional con bancos de capacitores facilita la propagación armónica. Esto es, la amplificación de tensión armónica debido al fenómeno de resonancia entre los capacitores shunt y las inductancias de la línea. El uso de filtros pasivos para atenuar dicho fenómeno, se ve afectado por los inconvenientes de pérdida de sintonía de los mismos, ante variaciones en los parámetros de los componentes y por cambios en la configuración tanto interna como externa de la red.

En los últimos años se está prestando mayor atención a diferentes soluciones activas [1]-[2]. Se han desarrollado varias investigaciones tendientes a mejorar la Calidad de Energía [3]-[6]. Entre todas las alternativas de compensación, la topología híbrida, con filtros activos y pasivos, resulta muy atractiva en plantas industriales donde alguna compensación pasiva ya está instalada [7]-[9]. La reconfiguración de la red ante fallas internas impone nuevas restricciones en diferentes subestaciones de la misma. Se han realizado estudios de armónicas considerando la configuración actual y futura de la red, verificando las distorsiones de tensión en las barras de $33 \mathrm{kV}$ de la planta y las condiciones de funcionamiento de los bancos de capacitores.

En este trabajo, se presenta una topología activa híbrida conectada en el nivel de MT (33kV) para solucionar el problema de compensación de potencia reactiva $y$ distorsiones armónicas. Se propone como algoritmo de control el marco de referencia sincrónico (d-q) y un sistema de control realimentado (feedback).

\section{DESCRIPCIÓN DE LA PLANTA INDUSTRIAL}

\section{A. Datos de la red}

La Fig. 1 muestra el circuito unifilar simplificado del modelo de red adoptado para realizar el estudio. Los datos de la red fueron presentados en [8].

Actualmente, con el objetivo de mejorar los perfiles de tensión de las barras de la planta y lograr un dado factor de potencia en el punto común de acoplamiento (PCA) al sistema en $132 \mathrm{kV}$, la planta posee dos bancos de capacitores para la compensación de reactivo de $7 \mathrm{MVAr} \mathrm{c} / \mathrm{u}$, conectados en nodos de $33 \mathrm{kV}$ tal como se muestra en la Fig.1, totalizando $\mathrm{Q}_{\mathrm{C}}=14 \mathrm{MVAr}$. Basados en estudios de flujos de carga, la demanda total considerada en este modelo en el PCA es $\mathrm{P} \cong 50 \mathrm{MW}, \cos \varphi=0.8$ ( $\left.\mathrm{Q}_{\mathrm{C}}=0 \mathrm{MVAr}\right) \mathrm{y}$ $\cos \varphi=0.9\left(\mathrm{Q}_{\mathrm{C}}=14 \mathrm{MVAr}\right)$. 


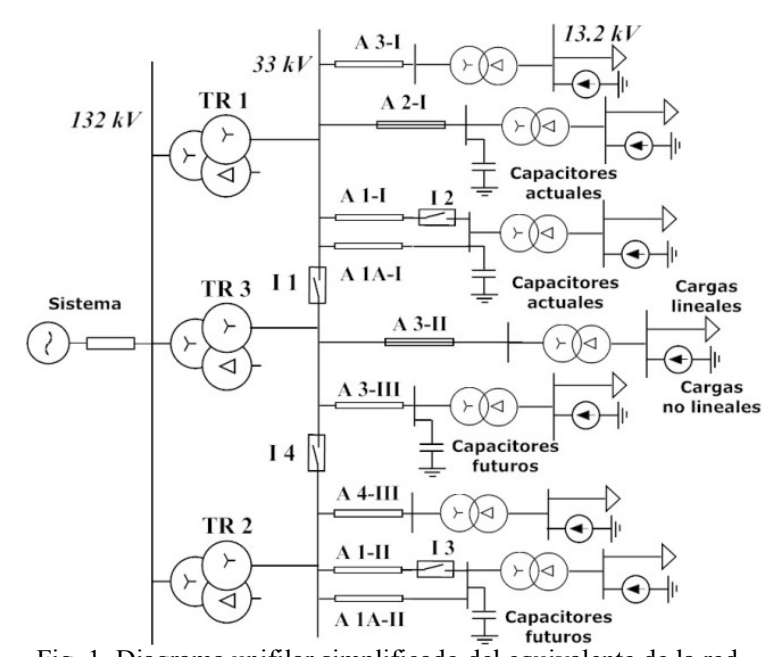

Fig. 1. Diagrama unifilar simplificado del equivalente de la red.

Debido a problemas de capacidad del equipamiento de maniobra instalado, ante ciertas fallas en la red interna de la planta, se analizó en [8] el escenario con la apertura del interruptor I1. En este trabajo, además se analiza la apertura del interruptor I4 mostrado en la Fig. 1. Esto define distintos escenarios postfalla diferentes a los presentados en [8], los cuales serán presentados y analizados más adelante. Los registros de mediciones y los valores de las armónicas, para una de las condiciones postfalla de la red fueron presentados en [8]. La demanda postfalla resultó menor que la prefalla.

En el futuro, con el incremento de un $20 \%$ de la demanda de la planta, los requerimientos de mejora de los perfiles de tensión en el nivel de $33 \mathrm{kV}$, como así también seguir manteniendo un determinado factor de potencia en el PCA, establecen la necesidad de aumentar la compensación de reactivo [8]. Dicha compensación futura, conectada en los puntos de $33 \mathrm{kV}$ tal como se muestra en la Fig.1, establece resonancias paralelo las cuales incrementan las armónicas a niveles inaceptables [8].

\section{CONFIGURACIONES OPERATIVAS DE LA Planta. DEFINICIÓN DE ESCENARIOS}

\section{A. Configuración de operación normal. Escenario I}

La configuración de operación normal de la planta, es la configuración prefalla, con los tres transformadores TR1, TR2 y TR3 conectados en paralelo en $33 \mathrm{kV}$, ver Fig. 1. Este escenario se define como el Escenario I.

\section{B. Posibles configuraciones de operación postfalla. \\ Distribución de carga original. Escenarios II y III}

Existe la posibilidad que ante fallas internas en la red, abra el interruptor I1, estableciendo un escenario postfalla, donde operan en paralelo en $33 \mathrm{kV}$ el TR3 y TR2 alimentando ciertas cargas, y el TR1 alimentando otras cargas. La demanda postfalla resultó menor que la prefalla, quedando conectados al TR1 los alimentadores A3-I, A2-I, A1-I y A1A-I, y el resto al TR2 y TR3, ver Fig. 1. Este escenario se define como el Escenario II.

Además, ante fallas internas también podrían abrir ambos interruptores I1 e I4, quedando las tres barras de $33 \mathrm{kV}$ de los TRs separadas. En ese caso, se consideraron los mismos alimentadores del escenario prefalla conectados al TR1, los alimentadores A3-II y A3-III conectados al TR3 y los alimentadores A4-III, A1-II y 1A-II, conectados al TR2, ver Fig. 1. Este escenario se define como el Escenario III.

\section{Posibles configuraciones de operación postfalla. Distribución de carga diferente. Escenarios $I V y V$}

Cabe aclarar, que en la planta existe la posibilidad de alimentar las cargas en $13.2 \mathrm{kV}$, de forma tal de abastecer las mismas desde las barras de $33 \mathrm{kV}$ correspondientes al TR1 o al TR2 o al TR3. Para un determinado escenario postfalla, presumiblemente las mayores necesidades de compensación aparecerán en las barras de $33 \mathrm{kV}$ con mayor carga.

A partir del Escenario II (I1 abierto e I4 cerrado), se considera otra distribución de cargas en 13.2kV. Se consideraron los alimentadores A3-I, A2-I, A1-I y A1A-I, antes conectados al TR1, ahora conectados al TR2 y TR3, ver Fig. 1. El resto de alimentadores antes conectados al TR2 y TR3, alimentadores A3-II y A3-III y A4-III, A1-II y 1A-II, ahora conectados al TR1. O sea, se intercambiaron las cargas del TR1 con las del TR2 y TR3. Este escenario se define como el Escenario IV.

A partir del Escenario III (I1 abierto e I4 abierto), se considera otra distribución de cargas en $13.2 \mathrm{kV}$. Se consideraron los alimentadores A3-I, A2-I, A1-I y A1A-I, antes conectados al TR1, ahora conectados al TR3, ver Fig. 1. Los alimentadores antes conectados al TR3, alimentadores A3-II y A3-III, ahora conectados al TR1 y se mantienen los alimentadores A4-III, A1-II y 1A-II, conectados al TR2. Este se define como el Escenario V.

\section{IV.FILTRO ACTIVO HÍBRIDO SHUNT-SERIE}

Una estructura general del Filtro Activo de Potencia Híbrido Shunt-Serie (FAPHS) se presenta en la Fig 2. En la Fig. 3, se presenta el sistema de control. La descripción del FAPHS y del sistema de control fue realizada en [8].

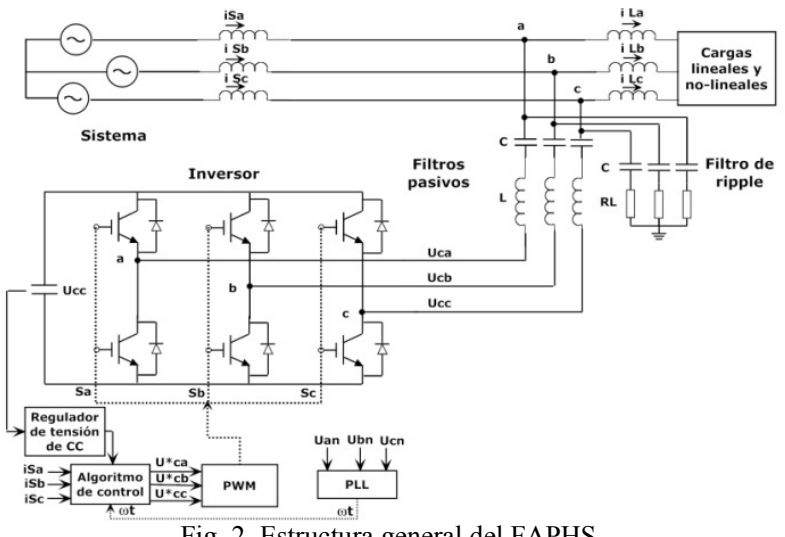

Fig. 2. Estructura general del FAPHS

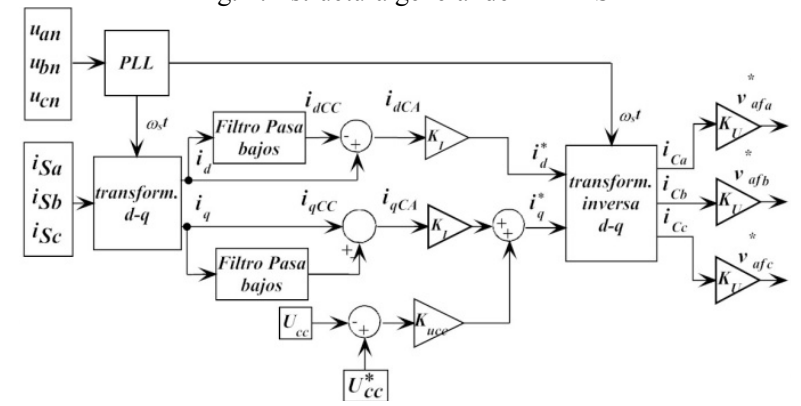

Fig. 3. Diagrama en bloques del control

El filtro activo es sólo utilizado para compensar las armónicas. Los filtros pasivos (FP) tiene dos funciones principales: compensación de reactivo y absorción de corrientes armónicas producidas por la carga. Es conveniente poseer varios niveles de compensación de potencia reactiva, con el fin de compensar dos estados de 
carga diferentes de la planta, como son el prefalla y el postfalla. El FP consiste de un filtro simple LC sintonizado a una determinada frecuencia y cierto $\mathrm{Q}_{\mathrm{C}}$ (MVAr) de potencia reactiva [12]. Se proponen dos filtros pasivos sintonizados a la $13^{\text {va }}$ armónica para proveer dos módulos de potencia reactiva. El FAPHS tiene la opción de conectar uno o dos de los FP a la salida del inversor, obteniendo así dos módulos de compensación de reactivo.

El sistema de control mide las corrientes trifásicas del sistema $\left(\mathrm{i}_{\mathrm{Sa}}, \mathrm{i}_{\mathrm{Sb}}, \mathrm{i}_{\mathrm{Sc}}\right)$, las tensiones trifásicas $\left(\mathrm{V}_{\mathrm{an}}, \mathrm{V}_{\mathrm{bn}}, \mathrm{V}_{\mathrm{cn}}\right) \mathrm{y}$ la tensión de $\mathrm{CC}$ del inversor para construir las referencias para el FAPHS. Utiliza la transformación (d-q), a un sistema rotante sincrónico con la tensión de secuencia positiva del sistema, provista por un PLL [9].

\section{AltERnATIVAS DE COMPENSACIÓN CON EL FAPHS}

En este trabajo, se considera la siguiente alternativa.

Alternativa de Compensación con un sólo FAPHS: se requiere medición y equipamiento para permitir la conexión en cualquiera de los tres TRs (TR1, TR2 y TR3) en $33 \mathrm{kV}$.

El criterio adoptado en este trabajo será conectar el FAPHS al transformador donde se registren las mayores corrientes. En [8], el FAPHS siempre fue conectado en el TR1, ya que no se analizaron los escenarios IV y V definidos en este trabajo los cuales contemplan distribuciones de carga y corrientes diferentes por los TRs.

Siendo el flujo de reactivo por cada TR no necesariamente el mismo, se deberá verificar si la compensación es adecuada, primordialmente evitando la sobrecompensación de reactivo, ya que la compensación por parte del FAPHS es fija y depende del diseño de los FP.

\section{RESULTADOS DE LA COMPENSACIÓN}

El modelo digital de la red y la topología de filtro propuesta para compensar en la planta bajo estudio es implementado para su evaluación en MATLAB/Simulink Power System Blockset (PSB) y los resultados son presentados en esta sección.

\section{A. Escenario I-FAPHS conectado en TR3}

En este caso, al estar las tres barras de $33 \mathrm{kV}$ de los TRs en paralelo, los resultados de las distorsiones en $33 \mathrm{kV}$, son independientes de donde se considere la ubicación de las cargas distorsionantes en $13.2 \mathrm{kV}$. Los valores de las potencias de carga en $33 \mathrm{kV}$ de cada TR son: $\mathrm{P}_{\mathrm{TR} 1}=17.4 \mathrm{MW}$, $\mathrm{Q}_{\mathrm{TR} 1}=12.5 \mathrm{MVAr}, \quad \mathrm{P}_{\mathrm{TR} 2}=17.4 \mathrm{MW}, \quad \mathrm{Q}_{\mathrm{TR} 2}=12.5 \mathrm{MVAr}$, $\mathrm{P}_{\mathrm{TR} 3}=26.4 \mathrm{MW}, \mathrm{Q}_{\mathrm{TR} 3}=19 \mathrm{MVAr}$, resultando una carga total en $33 \mathrm{kV}$ de: $\mathrm{P}_{\text {total }}=61.2 \mathrm{MW}, \mathrm{Q}_{\text {total }}=44 \mathrm{MVAr}$. Se observa que al ser TR1 y TR2 de similares características y de 63MVA y el TR3 de 90MVA, al estar conectados en paralelo, la distribución de cargas por el TR1 y TR2 es la misma y por el TR3 es mayor al resto. Debido a ello, se determinó la medición y conexión del FAPHS en su conjunto, inversor y filtros pasivos, en la barra de $33 \mathrm{kV}$ del TR3 (ver Fig. 1).

Se consideró la compensación total de reactivo de 30MVAr y resultó un diseño del FAPHS con $U_{c c}=1500 \mathrm{~V}$ y $\mathrm{C}=3000 \mu \mathrm{F}$. Los parámetros de diseño de los FP son: $\mathrm{Q}_{\mathrm{C}}=30 \mathrm{MVAr}$ totales, frecuencia de sintonía $\mathrm{f}_{\mathrm{s}}=650 \mathrm{~Hz}$, factor de calidad $\mathrm{Q}=45$.

La Fig. 4(a) muestra las formas de onda de la tensión y corriente de fase en la barra de $33 \mathrm{kV}$ del TR3, mientras que la Fig. 4(b) presenta las armónicas y el THD.

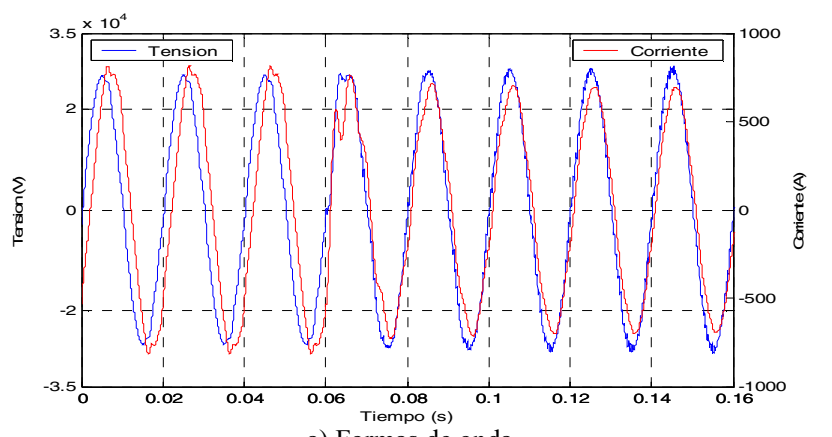

a) Formas de onda

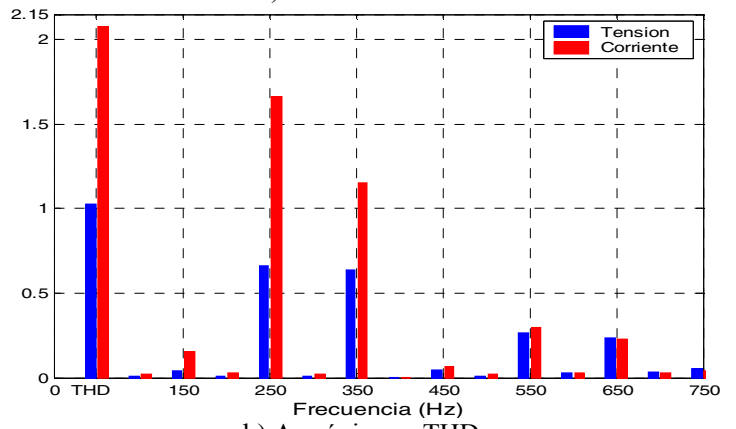

b) Armónicas y THD

Fig. 4. Escenario I. Tensión y corriente en 33kV del TR3.

En todos los casos, las componentes armónicas de la tensión y la corriente, son las correspondientes al estado estacionario final de compensación. Las componentes armónicas y los valores de $\mathrm{THD}_{\mathrm{U}} \mathrm{y} \mathrm{THD}_{\mathrm{I}}$, son expresadas en porcentaje del valor de la componente de $50 \mathrm{~Hz}$.

Los resultados de la Fig. 4 muestran un decrecimiento de las armónicas de tensión y corriente y por ende de los $\mathrm{THD}_{\mathrm{U}}$ y $\mathrm{THD}_{\mathrm{I}}$ con respecto a los casos de compensación con bancos de capacitores, presentados en [8]. Los resultados de las distorsiones armónicas de las tensiones y corrientes en $33 \mathrm{kV}$ son iguales en los tres TRs.

La Fig. 5(a) muestra la forma de onda de la corriente de una fase inyectada por el FAPHS en $33 \mathrm{kV}$, mientras que la Fig. 5(b) muestra su espectro en frecuencia (valores pico).

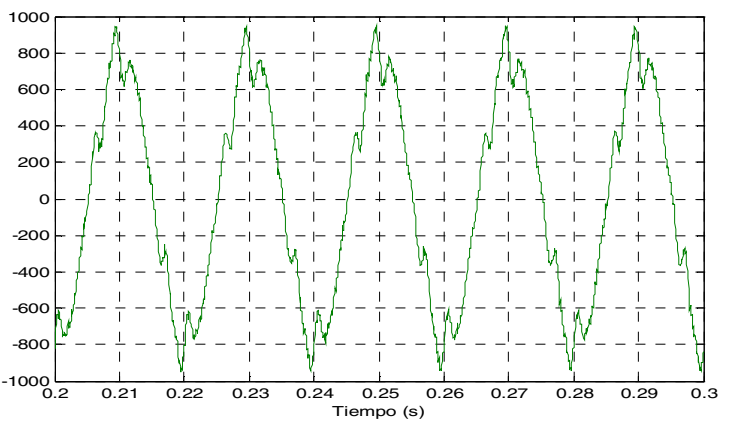

a) Formas de onda

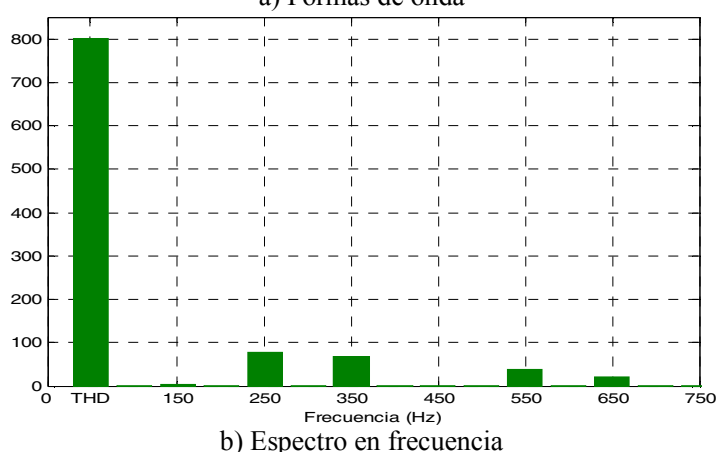

Fig. 5. Escenario I. Corriente inyectada por el FAPHS en $33 \mathrm{kV}$ 
Los resultados muestran que el valor fundamental corresponde a los 30MVAr de compensación de potencia reactiva. Respecto de las armónicas, el FP compensa la $13^{\text {va }}$ armónica y la parte activa compensa el resto. En la Tabla I se resumen los resultados de la compensación.

\section{B. Escenario IV-FAPHS conectado en TR3}

En este caso, al estar la barra de $33 \mathrm{kV}$ del TR1 separada de las barras de $33 \mathrm{kV}$ de los TR2 y TR3, las cuales se encuentran en paralelo, los resultados de las distorsiones en $33 \mathrm{kV}$, son dependientes de donde se considere la ubicación de las cargas distorsionantes en $13.2 \mathrm{kV}$.

La carga en cada TR en $33 \mathrm{kV}$ son: $\mathrm{P}_{\mathrm{TR} 1}=6.15 \mathrm{MW}$, $\mathrm{Q}_{\mathrm{TR} 1}=6.4 \mathrm{MVAr}, \quad \mathrm{P}_{\mathrm{TR} 2}=7.7 \mathrm{MW}, \quad \mathrm{Q}_{\mathrm{TR} 2}=6.1 \mathrm{MVAr}$, $\mathrm{P}_{\mathrm{TR} 3}=11.75 \mathrm{MW}, \mathrm{Q}_{\mathrm{TR} 3}=9.3 \mathrm{MVAr}$. Por ende, resulta una carga total en $33 \mathrm{kV}$ de: $\mathrm{P}_{\text {total }}=25.6 \mathrm{MW}, \mathrm{Q}_{\text {total }}=21.8 \mathrm{MVAr}$.

Dado que por el TR3 la carga es mayor, se determinó la medición y conexión del FAPHS en su conjunto, inversor y filtros pasivos, en la barra de $33 \mathrm{kV}$ del TR3 (ver Fig. 1).

Se utilizó el mismo FAPHS diseñado para el Escenario I. Se determinó una compensación de reactivo máxima de $13 \mathrm{MVAr}$, con la cual no se sobrecompensa el reactivo demandado por la carga total en $33 \mathrm{kV}$ del TR2 y TR3 y se alcanzan los requisitos en las barras de $33 \mathrm{kV}$ y $132 \mathrm{kV}$. El parámetro de diseño del FP es: módulo de $\mathrm{Q}_{\mathrm{C}}=13 \mathrm{MVAr}$, $\mathrm{f}_{\mathrm{s}}(\mathrm{Hz})=650, \mathrm{Q}=45$ (factor de calidad). Del análisis de este Escenario IV y del Escenario I, surge el diseño de dos módulos del FP, uno de 13MVAr y otro de 17MVAr, para completar los 30MVAr totales.

La Fig. 6(a) muestra las formas de onda de la tensión y corriente de fase en la barra de $33 \mathrm{kV}$ del TR3 y TR2, mientras que la Fig. 6(b) presenta las armónicas conjuntamente con el THD.

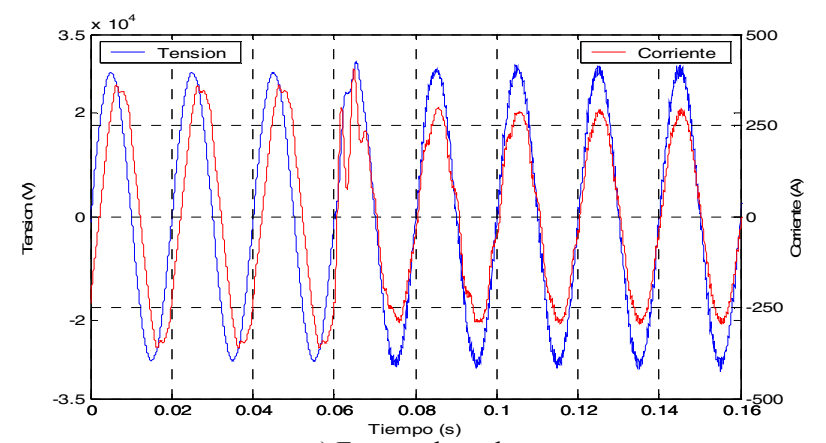

a) Formas de onda

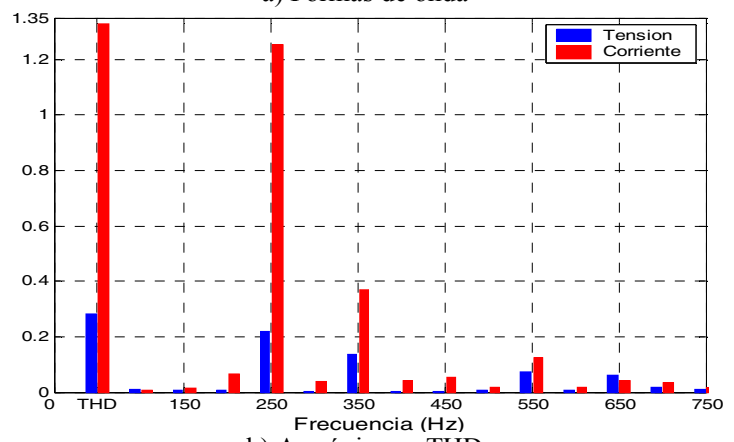

b) Armónicas y THD

Fig. 6. Escenario IV. Tensión y corriente en $33 \mathrm{kV}$ del TR3.

Los resultados de la Fig. 6 (a) muestran la compensación en el TR1 y un decrecimiento de las armónicas de tensión y corriente y por ende de los $\mathrm{THD}_{\mathrm{U}}$ y $\mathrm{THD}_{\mathrm{I}}$ con respecto a los casos de compensación con bancos de capacitores [8].
La Fig. 7 (a) muestra la forma de onda de la corriente de una fase inyectada por el FAPHS en $33 \mathrm{kV}$, mientras que la Fig. 7 (b) muestra su espectro en frecuencia (valores pico).

Se observa que el valor fundamental corresponde a los 13MVAr de compensación de reactivo. Las armónicas, provee aquellas que no son compensadas por el FP. En la Tabla I se resumen los resultados de la compensación.

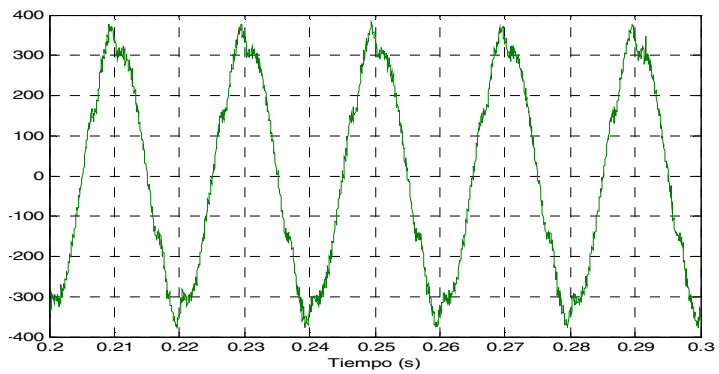

a) Forma de onda

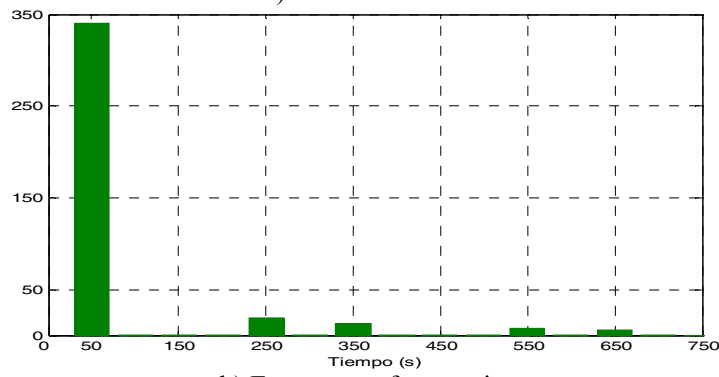

b) Espectro en frecuencia

Fig. 7. Escenario IV. Corriente inyectada por el FAPHS en $33 \mathrm{kV}$

\section{Escenario IV-FAPHS conectado en TRI}

Es el mismo escenario IV analizado anteriormente, donde se cambia el punto de medición y conexión del FAPHS a la barra de $33 \mathrm{kV}$ del TR1 (ver Fig. 1). Se utilizó el mismo FAPHS diseñado para el Escenario I.

Dado que el valor de potencia de carga en $33 \mathrm{kV}$ del TR1 es: $\mathrm{P}_{\mathrm{TR} 1}=6.15 \mathrm{MW}, \mathrm{Q}_{\mathrm{TR} 1}=6.4 \mathrm{MVAr}$, se determinó una compensación de reactivo máxima de $6 \mathrm{MVAr}$, con la cual no se sobrecompensa el reactivo demandado por la carga.

La Fig. 8(a) muestra las formas de onda de la tensión y corriente de fase en la barra de $33 \mathrm{kV}$ del TR1, mientras que la Fig. 8(b) presenta las armónicas y el THD.

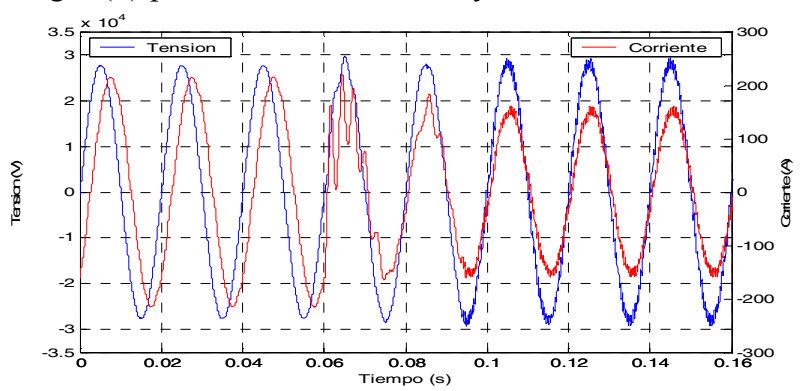

a) Formas de onda

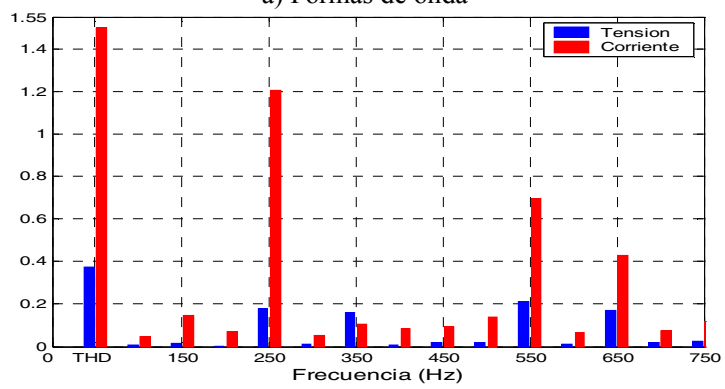

b) Armónicas y THD

Fig. 8. Escenario IV. Tensión y corriente en $33 \mathrm{kV}$ del TR1. 
Los resultados de la Fig. 8(a) muestran la compensación de reactivo en el TR1.

Las Figuras 9 y 10 muestran las formas de onda de la tensión y corriente de fase en las barras de $33 \mathrm{kV}$ del TR2 y TR3. Se observa la no variación de las formas de onda, ya que toda la compensación es realizada sólo en el TR1.

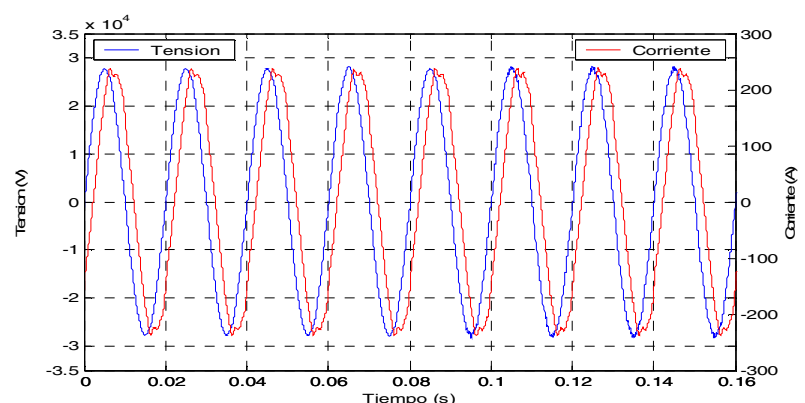

Fig. 9. Escenario V. Tensión y corriente en $33 \mathrm{kV}$ del TR3.

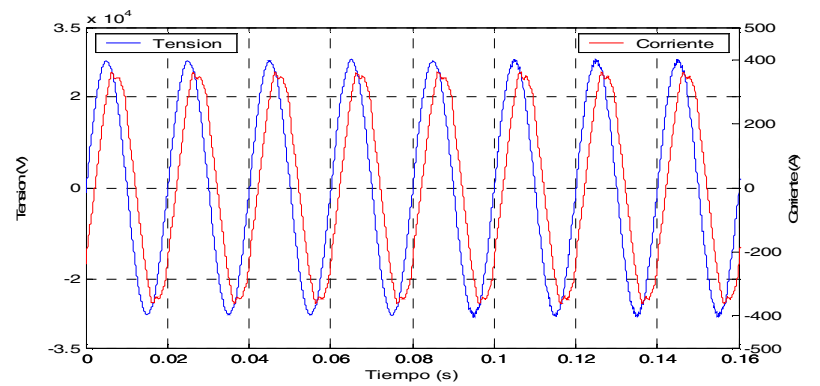

Fig. 10. Escenario V. Tensión y corriente en $33 \mathrm{kV}$ del TR2.

En la Tabla I se resumen los resultados. Se observa que la compensación de 6MVAr con el FAPHS en el TR1 es insuficiente para alcanzar el $\cos \varphi$ requerido en $132 \mathrm{kV}$.

\section{Escenario V-FAPHS conectado en TR3}

En este caso, al estar todas las barras de $33 \mathrm{kV}$ del TR1, TR2 y TR3, operando en forma separadas, los resultados de las distorsiones en $33 \mathrm{kV}$, son dependientes de donde se considere la ubicación de las cargas distorsionantes.

Los valores de potencias de carga en cada transformador en $33 \mathrm{kV}$ son: $\mathrm{P}_{\mathrm{TR} 1}=2.8 \mathrm{MW}, \mathrm{Q}_{\mathrm{TR} 1}=2.7 \mathrm{MVAr}, \mathrm{P}_{\mathrm{TR} 2}=3.5 \mathrm{MW}$, $\mathrm{Q}_{\mathrm{TR} 2}=3.65 \mathrm{MVAr}, \mathrm{P}_{\mathrm{TR} 3}=19 \mathrm{MW}, \mathrm{Q}_{\mathrm{TR} 3}=15.3 \mathrm{MVAr}$. Por ende, resulta una carga total en $33 \mathrm{kV}$ de: $P_{\text {total }}=25.3 \mathrm{MW}$, $\mathrm{Q}_{\text {total }}=21.7 \mathrm{MVAr}$.

Dado que por el TR3 la carga es mayor, se determinó la medición y conexión del FAPHS en su conjunto, inversor y filtros pasivos, en la barra de $33 \mathrm{kV}$ del TR3 (ver Fig. 1).

Habiendo definidos ambos módulos de compensación, se consideró una compensación de reactivo de 13MVAr, con la cual no se sobrecompensa la carga en $33 \mathrm{kV}$ del TR3.

La Fig. 11(a) muestra las formas de onda de la tensión y corriente de fase en la barra de $33 \mathrm{kV}$ del TR3, mientras que la Fig. 11(b) presenta las armónicas y el THD. La Fig. 12 muestra las formas de onda de la tensión y corriente de fase en la barra de $33 \mathrm{kV}$ del TR1. Se observa la no variación de las formas de onda, ya que toda la compensación es realizada sólo en el TR3. En la Tabla I se resumen los resultados.

\section{E. Escenario $V$-FAPHS conectado en TR2}

Es el mismo escenario $\mathrm{V}$ analizado anteriormente, donde se cambia el punto de medición y conexión del FAPHS a la barra de $33 \mathrm{kV}$ del TR2 (ver Fig. 1). Se utilizó el mismo FAPHS diseñado para el Escenario I.

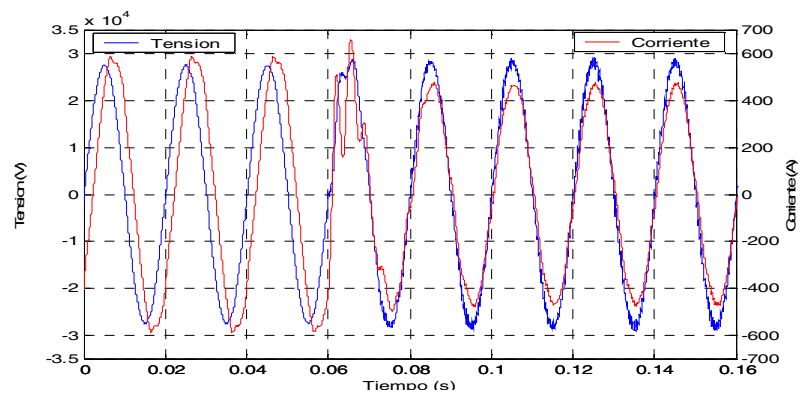

a) Formas de onda

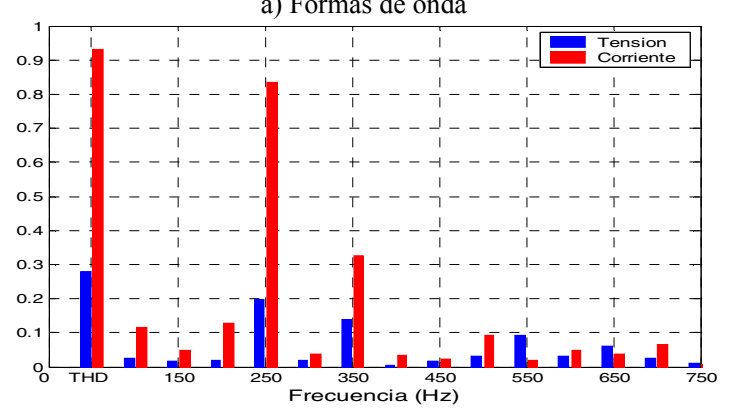

b) Armónicas y THD

Fig. 11. Escenario V. Tensión y corriente en $33 \mathrm{kV}$ del TR3.

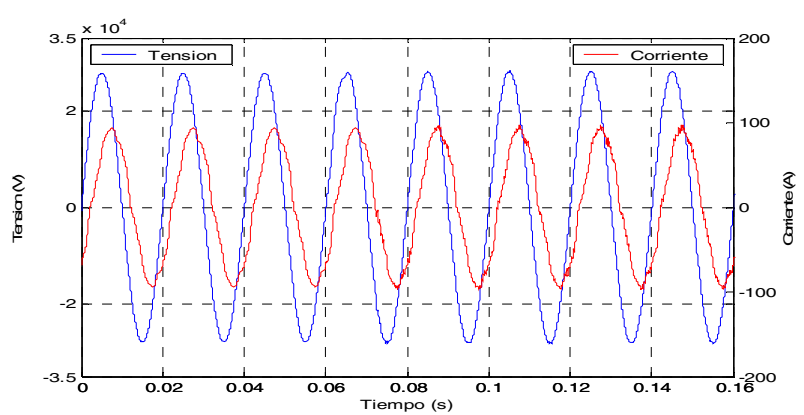

Fig. 12. Escenario V. Tensión y corriente en $33 \mathrm{kV}$ del TR1.

Dado que el valor de potencia de carga en $33 \mathrm{kV}$ del TR2 es: $\mathrm{P}_{\mathrm{TR} 2}=3.5 \mathrm{MW}, \mathrm{Q}_{\mathrm{TR} 2}=3.65 \mathrm{MVAr}$, se determinó una compensación de reactivo máxima de $3 \mathrm{MVAr}$, con la cual no se sobrecompensa el reactivo demandado por el TR1.

La Fig. 13(a) muestra las formas de onda de la tensión y corriente de fase en la barra de $33 \mathrm{kV}$ del TR2, mientras que la Fig. 13(b) presenta las armónicas y el THD.
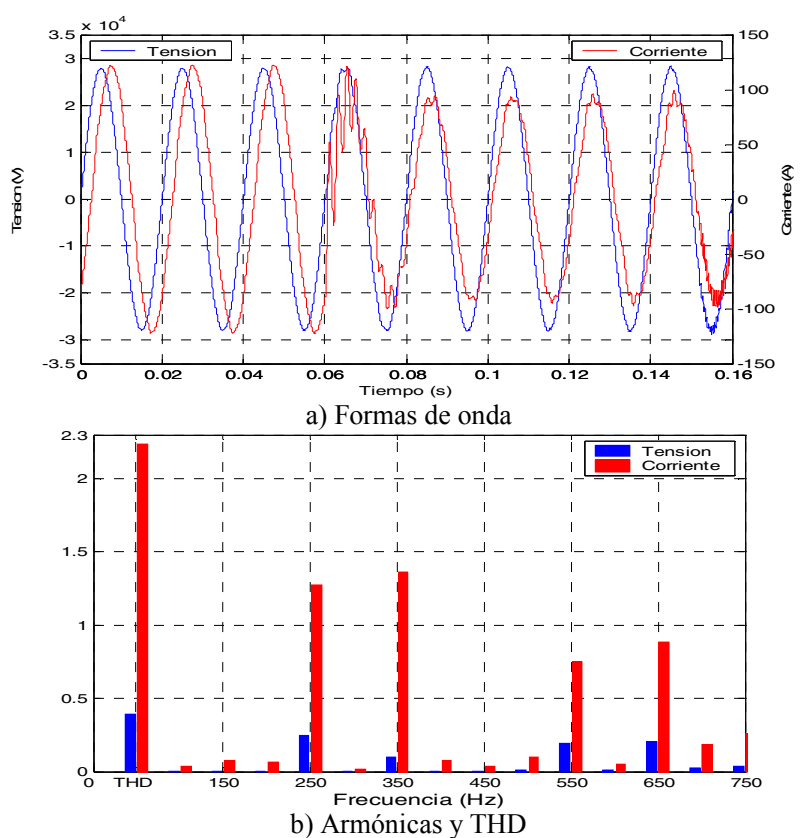

Fig. 13. Escenario V. Tensión y corriente en $33 \mathrm{kV}$ del TR2. 
Las Figuras 14 y 15 muestran las formas de onda de la tensión y corriente de fase en las barras de $33 \mathrm{kV}$ del TR1 y TR3. Se observa la no variación de las formas de onda, ya que toda la compensación es realizada sólo en el TR2.

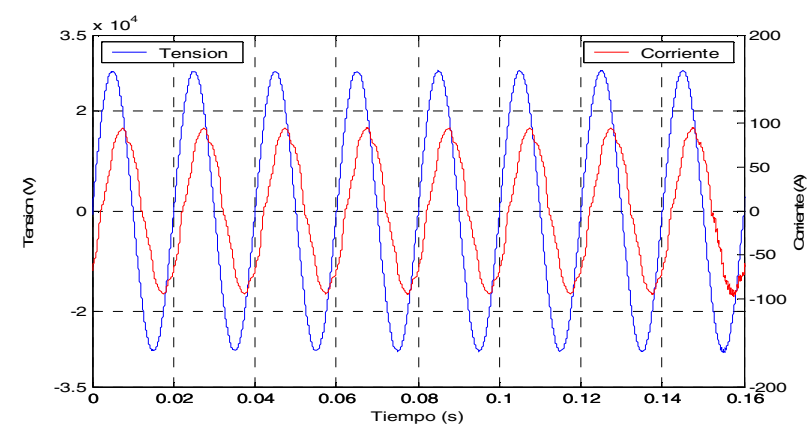

Fig. 14. Escenario V. Tensión y corriente en $33 \mathrm{kV}$ del TR1.

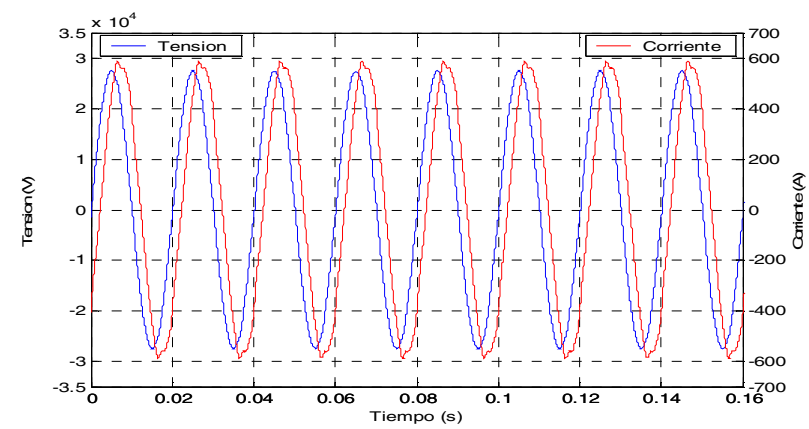

Fig. 15. Escenario V. Tensión y corriente en $33 \mathrm{kV}$ del TR3.

En la Tabla I se resumen los resultados. Se observa que la compensación en el escenario IV con el FAPHS conectado al TR1 compensando un máximo de 6MVAr y en el escenario $\mathrm{V}$ con el FAPHS conectado al TR2 compensando un máximo de $3 \mathrm{MVAr}$, resulta insuficiente para alcanzar el $\cos \varphi$ requerido en $132 \mathrm{kV}$.

TABLA I.

RESUMEN COMPENSACIÓN FAPHS

\begin{tabular}{|c|c|c|c|c|c|c|}
\hline $\begin{array}{c}\text { Escenario } \\
\text { Futuro }\end{array}$ & $\begin{array}{l}Q_{c} 33 k V \\
(M V A r)\end{array}$ & $\begin{array}{c}P 132 k V \\
(M W) \\
\end{array}$ & $\begin{array}{l}\text { Q132kV } \\
(M V A r)\end{array}$ & $P f_{t o t}$ & $\begin{array}{c}V_{\text {lfase }} 33 \mathrm{kV} \\
(\mathrm{pu})\end{array}$ & $\begin{array}{l}T H D_{V} \\
33 k V\end{array}$ \\
\hline $\begin{array}{c}\text { I } \\
\text { FAPHS TR3 }\end{array}$ & 30 & 66.1 & 16.6 & 0.97 & $\begin{array}{c}\text { TR1/TR2/TR3 } \\
1.03\end{array}$ & $1.18(\%)$ \\
\hline $\begin{array}{c}\text { II } \\
\text { FAPHS TR1 }\end{array}$ & 13 & 26.6 & 8.4 & 0.95 & $\begin{array}{c}\text { TR1 1.05 } \\
\text { TR2/TR3 } 1.05 \\
\end{array}$ & \begin{tabular}{|l|}
$0.72(\%)$ \\
$0.56(\%)$
\end{tabular} \\
\hline $\begin{array}{c}\text { III } \\
\text { FAPHS TR1 }\end{array}$ & 13 & 26.6 & 8.4 & 0.95 & $\begin{array}{ccc}\text { TR1 } & 1.05 \\
\text { TR2 } & 1.045 \\
\text { TR3 } & 1.05 \\
\end{array}$ & \begin{tabular}{|c|}
$0.6(\%)$ \\
$0.55(\%)$ \\
$0.58(\%)$ \\
\end{tabular} \\
\hline $\begin{array}{c}\text { IV } \\
\text { FAPHS TR3 }\end{array}$ & 13 & 27 & 9 & 0.95 & $\begin{array}{c}\text { TR1 } 1.038 \\
\text { TR2/TR3 } 1.05 \\
\end{array}$ & $\begin{array}{l}0.76(\%) \\
0.61(\%) \\
\end{array}$ \\
\hline $\begin{array}{c}\text { IV } \\
\text { FAPHS TR1 } \\
\end{array}$ & 6 & 26.4 & 17.7 & 0.83 & $\begin{array}{c}\text { TR1 } 1.05 \\
\text { TR2/TR3 } 1.03 \\
\end{array}$ & \begin{tabular}{|l|}
$0.65(\%)$ \\
$1.06(\%)$ \\
\end{tabular} \\
\hline $\begin{array}{c}\text { V } \\
\text { FAPHS TR3 }\end{array}$ & 13 & 26.7 & 9.2 & 0.95 & $\begin{array}{ccc}\text { TR1 } & 1.047 \\
\text { TR2 } & 1.045 \\
\text { TR3 } & 1.05\end{array}$ & $\begin{array}{l}0.71(\%) \\
0.55(\%) \\
0.59(\%) \\
\end{array}$ \\
\hline $\begin{array}{c}\text { V } \\
\text { FAPHS TR2 }\end{array}$ & 3 & 25.75 & 19.6 & 0.8 & $\begin{array}{lll}\text { TR1 } & 1.04 \\
\text { TR2 } & 1.05 \\
\text { TR3 } & 1.02\end{array}$ & $\begin{array}{l}0.79(\%) \\
0.68(\%) \\
1.23(\%)\end{array}$ \\
\hline
\end{tabular}

\section{CONCLUSIONES}

Se logró determinar el lugar apropiado de medición de las variables de referencias y punto de conexión del Filtro Activo de Potencia Hibrido, para cumplir con una compensación de reactivo y armónicas adecuada, en los diferentes escenarios más probables de operación de la red.

Se analizaron distintas variantes operativas de la red complementarias a las ya presentadas en [8], lo que contribuyó a definir un nuevo diseño del filtro pasivo. Resultaron dos módulos de compensación de reactivo, uno de 13MVAr y otro de 17MVAr, suponiendo que el filtro siempre se conectará al transformador con mayor carga. El diseño de los filtros pasivos, resultaron sintonizados en la armónica de orden 13.

También se analizan en este trabajo, escenarios postfalla en los cuales una vez abiertos el o los interruptores de $33 \mathrm{kV}$ el filtro se encuentra conectado al transformador con menor carga. En esta situación no se logra el factor de potencia deseado en el PCA, pero las distorsiones resultan aceptables en todas las barras de $33 \mathrm{kV}$.

El filtro activo hibrido presenta un desempeño adecuado, adaptándose a las distintas configuraciones de la red de la planta industrial considerada en este trabajo.

\section{REFERENCIAS}

[1] H. Akagi, E. Watanabe and M. Aredes, Instantaneous Power Theory and Applications to Power Conditioning, Series on Power Engineering, Piscataway, New Jersey: IEEE Press, 2007.

[2] T. Demirdelen, M. Inci, K.C. Bayindir, M. Tumay, "Review of hybrid active power filter topologies and controllers," in Proc. IEEE Power Engineering, Energy and Electrical Drives Conf., 2013, pp. 587-592.

[3] V.F. Corasaniti, M. Barbieri, P. Arnera and M. Valla, "Comparison of Active Filters Topologies in Medium Voltage Distribution Power Systems," in Proc. IEEE Power and Energy Society General Meeting, 2008, pp. 1-8.

[4] J.E. Hernandez, R.P. Kandula, F. Lambert, D. Divan, S. Grijalva, "A $7.2 \mathrm{kV}$ experimental setup of a third harmonic hybrid active filter for medium voltage utility applications," in Proc. IEEE Energy Conversion Congress and Exposition, 2013, pp. 5199-5206.

[5] W. Yen-Ching, L. Tzung-Lin, "A control method of hybrid filter for continuous reactive power compensation in a substation", in Proc. IEEE Applied Power Electronics Conference and Exposition, 2013, pp. 21862191.

[6] F. M. Muiño, C. E. D'Attellis y A. Carabio, "Estimación de Armónicos y Control de Filtros Activos," Décimo Tercer Encuentro Regional Iberoamericano del CIGRÉ (XIII ERIAC), 2009, trabajo C4-14.

[7] L. Herman, B. Blazic, I. Papic, "Performance of a parallel hybrid active filter with selective harmonic control," in Proc IEEE Power and Energy Society General Meeting, 2013, pp. 1-5.

[8] V. F. Corasaniti, M. B. Barbieri, P. L. Arnera, "Compensación con Filtro Activo de Potencia Hibrido en una Planta Industrial," Congreso Bienal de IEEE Argentina (ARGENCON), 2012, trabajo 085.

[9] V.F. Corasaniti, M.B. Barbieri, P.L. Arnera and M.I. Valla, "Hybrid power filter to enhance power quality in a medium voltage distribution network," IEEE Transactions on Industrial Electronics, vol. 56, no. 8, pp. 2885-2893, August 2009.

[10] Base Metodológica para el Control de la Calidad del Producto Técnico. Etapa 2. ANEXO a la Resolución ENRE 184/00, 2000.

[11] IEEE Recommended Practices and Requirements for Harmonic Control in Electrical Power Systems, IEEE Std. 519-1992.

[12] J. Arrillaga y N. R. Watson, Power System Harmonics, 2nd ed., John Wiley \& Sons, Ltd, 2003. 